\title{
Multimarker approach in cardiovascular risk prediction
}

\author{
Ignatios Ikonomidis*, Christos A. Michalakeas, John Lekakis, Ioannis Paraskevaidis and
}

Dimitrios Th. Kremastinos

2nd Cardiology Department, University of Athens, Attikon Hospital, Athens, Greece

\begin{abstract}
Various biomarkers express different pathways and pathophysiologic mechanisms of cardiovascular disease, such as inflammation, oxidative stress, myocardial injury, activation of the neurohormonal pathways, myocardial stress and renal function. Current thinking supports the notion that the combination of these biomarkers could increase their diagnostic and prognostic value. The multimarker approach offers benefits since it increases the diagnostic and prognostic information and may help in the design of a strategy for prevention or management of cardiovascular diseases. The purpose of the current review is to describe the characteristics of promising biomarkers which have shown an important additive value in the assessment of cardiovascular risk. Also, an extended reference is made regarding studies that address the prognostic value of multimarker models in the settings of primary prevention of cardiovascular disease and secondary prevention for patients with acute coronary syndromes, chronic coronary artery disease and heart failure.
\end{abstract}

Keywords: Multimarker, biomarkers, cardiovascular risk, coronary artery disease, heart failure

\section{Abbreviations and acronyms}

$\begin{array}{ll}\text { ACS: } & \text { Acute Coronary Syndrome } \\ \text { ADM: } & \text { Adrenomedullin } \\ \text { BNP: } & \text { Brain Natriuretic Peptide } \\ \text { CAD: } & \text { Coronary Artery Disease } \\ \text { CRP: } & \text { C-Reactive Protein } \\ \text { cTnT, cTnI: } & \text { Cardiac Troponins T and I } \\ \text { ET-1: } & \text { Endothelin-1 } \\ \text { IL-1b, IL-6, IL-10: } & \text { Interleukin 1b, Interleukin- } \\ & \text { 6, Interleukin-10 } \\ \text { Lp-PLA } 2: & \text { Lipoprotein-associated } \\ & \text { Phospholipase A } 2 \\ \text { MCSF: } & \text { Monocyte/Macrophage } \\ & \text { Colony Stimulating Factor } \\ \text { MPO: } & \text { Myeloperoxidase }\end{array}$

${ }^{*}$ Corresponding author: Dr. Ignatios Ikonomidis, MD, FESC, 2nd Cardiology Department, University of Athens, Attikon Hospital, Perikleous 19, N. Chalkidona, Athens 14343, Greece. Tel.: +30 694 4805732; Fax: +30 210 5832351; E-mail: ignoik@otenet.gr.

$\begin{array}{ll}\text { MR-pro-ADM: } & \begin{array}{l}\text { Midregional } \\ \text { pro-Adrenomedullin } \\ \text { Amino-terminal pro Brain } \\ \text { NT-Pro-BNP: }\end{array} \\ & \begin{array}{l}\text { Natriuretic Peptide } \\ \text { Osteoprotegerin }\end{array} \\ \text { OPG: } & \text { Transforming Growth Factor } \\ \text { TGF } \beta-1: & \beta-1 \\ \text { TNF- } \alpha: & \text { Tumor Necrosis Factor } \alpha \\ \text { WBC: } & \text { White Blood Cell Count } \\ \text { WBCHO: } & \text { Whole Blood Choline }\end{array}$

\section{Introduction}

Circulating biomarkers have long since become a daily tool for the clinician in the diagnosis, management and prognosis of cardiovascular disease. In this context, novel biomarkers are continuously being developed in order to aid the physician in clinical decision and to enlighten pathophysiological paths associated with the pathogenesis of cardiovascular diseases and offer new targets for treatment. Different mechanisms, such as inflammation, oxidative stress, myocardial in- 
jury and cell apoptosis, activation of the neurohormonal pathways, elevation of intracardiac pressures and renal function, play important role in the pathogenesis of cardiovascular disease. Understanding of the above mechanisms may help in predicting the course of an acute cardiovascular event or the prognosis of a chronic ongoing disease process, and also aid in the design of a therapeutic strategy. It has been proposed that a multimarker approach to cardiovascular disease offers benefits in the assessment of cardiovascular risk, both for primary and secondary risk stratification. The purpose of the current review is: a) to describe the characteristics of promising biomarkers which have shown an important additive value in the assessment of cardiovascular risk, b) to review studies addressing the prognostic value of multimarker models in the settings of primary and secondary prevention of cardiovascular disease.

\subsection{Biomarkers of inflammation}

Inflammation is considered one of the major factors contributing to the pathogenesis of atherosclerosis [1]. A triggering effect, usually increased concentration of lipoproteins, but also shear stress forces, increased oxidative stress, angiotensin II, infections, initiates the atherogenic process. After the initial insult/injury of the vascular endothelium the process of atherosclerosis is perpetuated via inflammatory mechanisms, which include the release of proinflammatory cytokines, such as monocyte/macrophage colony stimulating factor (MCSF), interleukin 1b (IL-1b) and interleukin-6 (IL6). These cytokines induce monocyte/macrophage activation, uptake of low density lipoprotein (LDL) and formation of foam cells which form the core of the atherosclerotic plaque. MCSF promotes the release of other cytokines such as IL-1b and IL-6 and finally leads to CRP production by the liver [2]. Furthermore, MCSF induces the production of monocyte chemoattractant protein-1 (MCP-1), which leads to leukocyte adhesion and migration into the vascular wall, and tissue factor (TF) related to plaque instability and thrombosis. Other cytokines such as TGF-1b have an antiinflammatory/atheroprotective action by strengthening the fibrous cap over the atherosclerotic plaque.

The White Blood Cell Count (WBC) is the most easily obtained marker of inflammation. A high WBC count has been associated with increased cardiovascular mortality and incidence of coronary artery disease (CAD) in apparently healthy adults. In the setting of a known cardiovascular disease, WBC count seems to correlate with increased risk for complications both in stable CAD (CAPRIE trial) [3] and acute coronary syndromes (ACS). In ACS, WBC count seems to have an additive prognostic value to that of troponin regarding the extent of CAD, TIMI flow and myocardial perfusion grade during angiography and mortality rate [4].

C-Reactive Protein (CRP) is the final end product of various inflammatory processes in atherosclerosis and acute coronary syndromes. It may reflect the cumulative effect of local vascular inflammation, but it may also have a direct proinflammatory effect on the endothelium by increasing the proinflammatory iNOS (nitric oxide synthetase) and reducing the atheroprotective eNOS. Its prognostic value has been shown for both primary and secondary prevention of coronary artery disease. There is evidence to support the additive value of CRP to that of traditional risk factors in the assessment of cardiovascular risk in apparently healthy adults [5,6], especially regarding values of the very low $(<0.5 \mathrm{mg} / \mathrm{L})$ and very high range $(>10 \mathrm{mg} / \mathrm{L})$ [7]. In the setting of an ACS, CRP has an additive predictive value to that of troponin [8] and it has been shown to predict long term survival regardless of LDL levels $[9,10]$. In acute and chronic heart failure CRP is an independent predictor of adverse outcomes [11].

The levels of Monocyte/macrophage colony-stimulating Factor (MCSF) have been found to be elevated in both stable CAD and acute coronary syndromes. MCSF is directly related to myocardial ischemia during effort and to the angiographic extend of coronary lesions [12]. Furthermore, levels of MCSF were found to have predictive value regarding short-term and longterm cardiovascular events in these situations, independently of traditional risk factors and CRP levels [13]. In acute coronary syndromes MCSF is a predictor of in-hospital mortality and short term survival. In chronic coronary artery disease increased levels of MCSF $(>800 \mathrm{pg} / \mathrm{mL})$ and $\mathrm{CRP}(>2.5 \mathrm{mg} / \mathrm{L})$ were associated with a greater absolute and relative risk for future cardiovascular events, CRP, however, predicts cardiovascular events at a longer follow up period.

Tumor Necrosis Factor $\alpha$ (TNF- $\alpha$ ) levels are elevated in patients with heart failure [14]. By activating matrix metalloproteinases TNF- $\alpha$ causes left ventricular dilatation and dysfunction. Moreover, as far as coronary artery disease is concerned, TNF- $\alpha$ and CRP retain their prognostic value independently of each other. However, TNF- $\alpha$ predicts short-term cardiovascular events in contrast to CRP which provides prognostic information for long-term outcomes [15]. Osteoprotegerin (OPG) is a member of the tumor necrosis 
factor receptor family. OPG levels correlate with the severity of coronary artery disease [16] and it has been found to be an independent risk factor for cardiovascular events [17].

Interleukin-6 (IL-6) levels are found to be increased in stable and acute coronary syndromes [18]. IL-6 has a negative inotropic action [19] and its levels are also associated with elevated intracardiac pressures in patients with ischemic cardiomyopathy [20]. IL-6 was found to be increased in response to reversible ischemia during dobutamine stress echo and was related to the extent of new wall motion abnormalities at peak stress [21]. Thus, an increase of IL- 6 at peak stress was a marker of reversible ischemia. Furthermore, increased IL-6 levels during recovery were related with a prolongation of the time to recovery of the new wall motion abnormalities observed at peak dobutamine stress echocardiography. This finding suggests that IL-6 may contribute to of left ventricular dysfunction and a may serve as predictor of the development of heart failure in patients with CAD. Indeed, changes of IL-6 during dobutamine stress echocardiography had an incremental value over wall motion abnormalities for the prediction of adverse outcome in CAD patients during a 6 year of followup [22].

Lipoprotein-associated Phospholipase $\mathrm{A}_{2}\left(\mathrm{Lp}-\mathrm{PLA}_{2}\right)$ is produced by inflammatory cells that are involved in the process of atherogenesis and is detected in human atherosclerotic lesions. Elevated Lp-PLA 2 levels are associated with an increased risk of cardiovascular events [23] and adverse cardiovascular events in patients with coronary artery disease independently of traditional risk factors and CRP levels [24]. Pharmaceutical interventions to reduce Lp-PLA 2 activity may result in additional anti-inflammatory effect in patients with atherosclerosis, as was shown for darapladib administration and its effect on reducing high sensitivity CRP and IL-6 levels [25].

There are cytokines whose actions in innate and adaptive immunity have an anti-inflammatory effect, and are thus considered to be atheroprotective. Interleukin-10 (IL-10) is secreted from activated macrophages and forces CD4+ cells to differentiate into Th2, a response which is considered atheroprotective [26], in contrast to IL-12 which promotes the atherogenic Th1 response. Similarly to IL-10, transforming growth factor $\beta-1$ (TGF $\beta-1)$ has an atheroprotective effect by inhibiting T-cell mediated immunity [27]. TGF $\beta-1$ also inhibits leucocyte adhesion molecules and MCP-1 expression, as well as smooth muscle cell differentiation, proliferation and migra- tion [28]. TGF- $\beta$ stimulates collagen production by smooth muscle cells leading to strengthening of the fibrous cap over the atheromatic plaque [29], and protecting from plaque instability and rapture.

\subsection{Biomarkers of oxidative stress}

Increased oxidative stress causes endothelial dysfunction, contributes to atherogenesis and leads to progression of heart failure through cell apoptosis and necrosis. Inflammation is tightly linked to oxidative stress status since various cytokines, such as interleukin-1, promote the release of superoxide anion. IL-1 also causes the release of $\mathrm{NO}^{-}$which reacts with the superoxide anion to form the peroxynitrite, leading to the formation of nitrotyrosine, a marker of nitrosative stress [30]. Nitrooxidative stress also has a deleterious effect on endothelial function and is responsible for abnormal coronary vasoactivity in experimental models.

Myeloperoxidase (MPO) is a hemoprotein expressed in neutrophils and secreted during their activation. It causes oxidative transformation of LDL particles and thus plays a role in the initiation and progression of atherosclerosis and cardiovascular disease. MPO has potent proinflammatory properties and may contribute to tissue injury. Plasma myeloperoxidase serves as an indirect marker of oxidative stress. MPO levels have been associated with the risk of coronary artery disease independently of other risk factors [31]. Its levels were found increased in patients with coronary artery disease [32] and it is proposed to have prognostic value in acute coronary syndromes [33]. MPO levels also correlate with the severity of heart failure [34] and have prognostic value for the survival of these patients.

\subsection{Biomarkers of myocardial injury}

Cardiac troponins $\mathrm{T}$ and $\mathrm{I}$ (cTnT, cTnI) are highly specific and sensitive markers of myocardial necrosis. It has been shown that cTn measurement as a diagnostic tool is more useful than older biomarkers of myocardial necrosis (CK-MB, myoglobulin). Even mild increase in troponin levels is of prognostic value in the setting of an acute coronary syndrome [35].

Other causes than ischemia may increase the levels of cardiac troponins. cTn may be increased in patients with heart failure without ischemia [36]. Myocardial injury and myocyte cell death can occur in heart failure due to elevated intracardiac pressures, inflammation or increased oxidative stress, and this fact, reflected on troponin levels, correlates with the severity of heart 
failure and its adverse effects. A level of $0.04 \mathrm{ng} / \mathrm{mL}$ was found to be the cutoff point for cTnI regarding survival in patients with advanced heart failure after adjustment for other predictors [37]. Hudson et al. reported that cTnT levels of more than $0.02 \mathrm{ng} / \mathrm{mL}$ were associated with a 4-fold risk for death in patients with chronic heart failure [38]. In acute decompensated heart failure the levels of cardiac troponin may be used as predictors of outcome [39]. As more sensitive assays for the detection of troponin levels are being developed, its role in the prognosis of patients with heart failure will become more important in the future.

\subsection{Biomarkers of increased myocardial stress - elevated intracardiac pressures}

The natriuretic peptides, brain natriuretic peptide (BNP) and amino-terminal pro brain natriuretic peptide (NT-Pro-BNP), are produced by the prohormone BNP which is released as a response to increased wall tension, dilatation or hypertrophy of the ventricles [40]. BNP is a bioactive peptide which causes arterial vasodilation, diuresis and natriuresis, whereas NT-Pro-BNP is inactive. However, NT-Pro-BNP has a longer biologic half-time period than BNP (approximately 120 minutes), a fact that makes its levels useful in the diagnosis of heart failure. As a diagnostic-screening tool, the cut-off levels of BNP and NT-Pro-BNP were estimated at $100 \mathrm{pg} / \mathrm{mL}$ and $340 \mathrm{pg} / \mathrm{mL}$ respectively, bearing in mind the limitations of measurements in special populations (i.e. the elderly, patients with renal insufficiency, obese patients, females). The levels of BNP correlate with the in-hospital mortality of patients with acutely decompensated heart failure [41] and can be used as a prognostic marker independently of other variables. In chronic heart failure, BNP also has prognostic value, and it can be used for the risk stratification of these patients [42]. Recent studies have proposed that BNP should be used as a guide to intensify therapy in heart failure patients. In the STARS-BNP trial BNP-guided therapy was superior in terms of survival compared to therapy guided by clinical criteria [43]. Logeart et al. proposed that predischarge BNP levels are a strong predictor for adverse outcomes and readmission of patients with decompensated heart failure [44].

Adrenomedullin (ADM) is produced as a response to increased intracardiac pressures or volume overload and causes vasodilation and natriuresis [45]. Its levels are higher in patients with heart failure and correlate with the severity of the disease [46]. Midregional pro-adrenomedullin (MR-pro-ADM) is a more stable molecule than adrenomedullin itself and can be measured more easily.

Increased levels of ST2, a member of the interleukin1 receptor family, were found in patients with severe heart failure. ST2 levels are strong predictors of mortality both in patients with acute coronary events [47] and heart failure [48]. It was recently reported that pharmaceutical inhibition of IL-1 in patients with rheumatoid arthritis by anakinra, an IL-1a receptor antagonist, improves vascular and left ventricular function and is associated with reduction of nitrooxidative stress and endothelin [49]. The same medication (anakinra) was found to ameliorate the remodelling process of the left ventricle by inhibiting cardiomyocyte apoptosis in experimental animal models with myocardial infarction [50].

\subsection{Biomarkers of neurohormonal activation}

The activation of the sympathetic nervous system and renin-angiotensin-aldosterone system (RAAS) has been tightly linked to the pathogenesis and progression of heart failure [51]. Plasma levels of norepinephrine are an independent predictor of mortality in patients suffering from heart failure and in patients with left ventricular systolic dysfunction. The levels of endothelin1 (ET-1) and big endothelin-1 correlate with the level of pulmonary artery pressure [52], and the severity and mortality of heart failure [53].

The activation of the RAAS is also prominent in acute coronary syndromes and leads to adverse cardiovascular effects via collagen synthesis and fibrosis. Reversal of this procedure by spironolactone, an inhibitor of aldosterone, reduces adverse left ventricular remodeling [54].

\subsection{Biomarkers of renal dysfunction}

Renal function is of pivotal importance in the assessment of cardiovascular events. Creatinine clearance is of additional prognostic value in the setting of an acute coronary event [55]. Cystatin $\mathrm{C}$ is a biomarker of renal function less influenced by gender, age and muscle mass than serum creatinine. Cystatin $\mathrm{C}$ correlates with the glomerular filtration rate which is generally accepted as an overall index of renal function. Cystatin $\mathrm{C}$ was found to be associated with peripheral atherosclerotic disease [56] and to predict the development of heart failure in the elderly [57]. Significant correlations were found between cystatin $\mathrm{C}$ values and other markers of cardiovascular disease, including CRP, in 
elderly men [58]. There are studies suggesting a prognostic role for cystatin $\mathrm{C}$ in heart failure [59] and acute coronary syndromes [60].

Microalbumiuria has been documented as a risk factor for future cardiovascular events in hypertensive patients. For every 10-fold increase in UACR (urine albumin-creatinine ratio), hazard ratios in nondiabetic patients increased as follows: cardiovascular mortality, by $97.7 \%$; all-cause mortality, by $75.2 \%$; stroke, by $51.0 \%$; and myocardial infarction, by $45 \%$ [61]. In patients with type 2 diabetes microalbuminuria and macroalbuminuria are considered risk factors for future cardiovascular events [62]. A significant percentage of patients with systolic heart failure without hypertension or diabetes also demonstrate microalbuminuria [63]. Ingelsson et al. have reported that microalbuminuria (defined as urinary albumin excretion rate of more than $4.7 \mu \mathrm{g} / \mathrm{min}$ ) is a marker of subclinical cardiovascular damage which may predispose for future development of heart failure in the elderly (hazard ratio: 1.49) [64].

\section{Multimarker risk prediction in various settings}

\subsection{Primary prevention}

Markers of inflammation have been used in the general population as predictors of cardiovascular disease, either on their own or in various combinations. White blood cell (WBC) count was shown to be a predictor of cardiovascular events and all-cause mortality in postmenopausal women, independently of CRP levels. Women in the fourth quartile of WBC count (670015000 cells/L) had over a 2 -fold elevated risk for coronary heart disease death (Hazard Ratio: 2.36), compared to women in the first quartile, after adjustment for known risk factors [65]. In the ABC study IL6 , CRP and TNF- $\alpha$ were examined regarding the incidence of cardiovascular events in the elderly. The three biomarkers were independently associated with the risk for developing heart failure and the greatest risk was found when all three biomarker measurements were in the top quartile [66]. Vasan et al. also reported that CRP, TNF- $\alpha$ and IL-6 levels could have prognostic value for the future development of heart failure in the elderly population. The combination of CRP with TNF- $\alpha$ seems to identify asymptomatic people for the risk of future development of heart failure in the Framingham Heart Study. Participants with elevated CRP levels ( $>5 \mathrm{mg} / \mathrm{dL}$ ) had a 3-fold increase of heart failure risk, and participants with increased levels of all three biomarkers had a 4-fold increase of risk [67]. The predictive value of IL-6 has been evaluated in several studies. It has been shown to be a risk factor for cardiovascular disease in apparently healthy men, independent of CRP [68]. However, IL-6's value as a prognostic marker in patients with acute coronary syndromes has not been established yet [69] and further studies are needed. On the other hand, CRP, IL-6 and other novel biomarkers where found to provide little additional prognostic information in a model including traditional risk factors in the ARIC study [70]. Ridker et al. in the recently published results of the JUPITER study, report that the use of hs-CRP levels as a guide to statin therapy in people without hyperlipidemia reduces the risk of adverse cardiovascular events in this apparently healthy population, suggesting an additive prognostic role of CRP to that of the already established role of LDL [71].

As far as markers of myocardial stress are concerned, the natriuretic peptides are the markers mostly studied. NT-Pro BNP was found to predict cardiovascular risk in nonhospitalised individuals aged 50-89 years [72]. In this study NT-pro-BNP was found to be a stronger biomarker in the prediction of cardiovascular events and death (Hazard Ratio:1.96) than CRP (Hazard Ratio:1.46) and urinary albumin/creatinine ratio (Hazard Ratio:1.88). In patients with other comorbidities such as hypertension, diabetes mellitus and asymptomatic coronary artery disease the value of BNP in predicting the risk for developing heart failure is also important. BNP may also be used to screen for cardiotoxic effects associated with the administration of certain medications [73].

In the HOPE study NT-Pro BNP was compared to traditional risk factors and other inflammatory biomarkers and it was found to be of greater value in the prediction of clinical outcome (myocardial infarction, stroke, cardiovascular death) during 4.5 years of follow-up than the 9 inflammatory biomarkers that were measured (hazard ratio for NT-Pro-BNP: 1.72 per increment SD, 95\% CI:1.39-2.12, $p<0.0001$ ) [74]. The result could be attributed to the significant day-to-day variability of inflammatory biomarker levels leading to misclassification of subjects, as was shown for CRP [75]. This can be rectified in part by the combination of more than one inflammatory biomarkers, as was done in the ABC [64] and FINRISK [13] studies.

CRP and NT-Pro-BNP may improve risk stratification of patients with chronic renal disease who present with increased levels of troponin. Patients with end stage renal disease and increased levels of cTnT and 
CRP had an increased all-cause mortality risk (HR:3 and HR:1.8 in the fourth quartile for cTnT and CRP respectively), during a mean period of 4 years [76]. In another study, Apple et al. found that hs-CRP, cTnI and cTnT have additive prognostic value for all cause death during a 2 year follow-up period in patients with end stage renal disease. Tertile analysis for NT-Pro-BNP also demonstrated prognostic value [77].

Wang et al. measured 10 biomarkers (C-reactive protein, BNP, NT-Pro-ANP, aldosterone, renin, fibrinogen, d-dimers, plasminogen-activator inhibitor type 1, homocysteine, urinary albumin-to-creatinine ratio) in a population of the Framingham Heart Study (3209 participants). During a median follow-up period of 7.4 years 207 participants died and 169 had a first major cardiovascular event (fatal or non-fatal myocardial infarction, prolonged angina, heart failure, stroke). Of all examined biomarkers, BNP, CRP, urinary albumin-tocreatinine ratio, homocysteine and renin levels added incremental information for the prediction of the overall mortality in this population. BNP and urinary albuminto-creatinine ratio showed the highest incremental predictive value for major cardiovascular events (adjusted HR:1.25 per $1 \mathrm{SD}$ increment in the log values for BNP and HR:1.20 per $1 \mathrm{SD}$ for urinary albumin-to-creatinine ratio). However, the use of multimarker scores was found to add only moderately to standard risk factors for risk assessment of individual persons (C statistic 0.77 with all predictors compared to 0.76 with age, sex and conventional risk factors as predictors) [78].

In a study by Zethelius et al., four biomarkers were measured (NT-Pro-BNP, cTnI, CRP, Cystatin-C) in the elderly (1135 participants, mean age at baseline 71 years) and there was a follow-up period of 10 years, during which 315 deaths from both cardiac and noncardiac causes were recorded. This multimarker strategy was found to add prognostic information to that of established risk factors for cardiovascular disease. The participants with elevated levels of two, three or all four biomarkers had a 3-, 7- or 16-fold risk for cardiovascular death respectively. The authors concluded that the incorporation of a combination of biomarkers that reflect myocardial damage, renal function, myocardial function and inflammation to a model with established risk factors improved risk stratification for cardiovascular mortality in the elderly population. This finding was attributed in part to the older age of their study population compared to previous studies, but also to the proper selection of those biomarkers that reflect independent pathophysiological mechanisms affecting outcome [79].

\subsection{Secondary prevention in}

\subsubsection{Coronary artery disease}

Acute Coronary Syndromes (ACS)

In acute coronary syndromes, white blood cell count seems to have an additive predictive value to that of troponin regarding the extent of CAD, TIMI flow and myocardial perfusion grade during angiography, and mortality rate. The combined use of CRP and WBC was proposed to stratify patients with ACS across an eightfold gradation of 6-month mortality risk [2]. In unstable angina MCSF has an additive prognostic value to that of CRP, independently of other traditional risk factors. As mentioned before, these biomarkers predict adverse cardiovascular events at a different stage in the depth of time, a fact that probably correlates with the production of these cytokines at different stages of the atherosclerotic process [11].

The addition of MCP-1 measurement to CRP and BNP levels provides additive prognostic value for adverse events (death, stroke, myocardial infarction, heart failure) in patients with acute coronary syndromes in the A to Z Study. A value of MCP-1 > $238 \mathrm{pg} / \mathrm{ml}$ was independently associated with mortality at 4 months of follow-up (HR: 1.76) [80]. In TACTICS-TIMI 18, patients with an ACS were found to have a 2.1-, 3.1, and 3.7-fold increase in the risk of death, myocardial infarction or heart failure in six months depending on the number of elevated biomarkers (CRP, BNP and cT$\mathrm{nI}$ ), after adjustment for known clinical predictors of outcome [81]. Also, CRP has been associated with IL-6, TNF- $\alpha$ and fibrinogen in the setting of an acute coronary event [82], and cardiac mortality was found to be 6-fold higher in patients in the highest tertile of CRP and IL-6 and 3-fold higher in patients in the highest tertile of fibrinogen and TNF- $\alpha$ compared to the lower tertiles respectively.

In The TACTICS-TIMI-18 study, women presenting with ACS with elevated CRP and BNP levels were found to benefit from early revascularization therapy even if cardiac troponin levels were normal [83]. This finding suggests that BNP and CRP may determine risk and thus, guide treatment in patients with normal troponin levels. Regarding other markers of myocardial necrosis, however, troponin alone was shown to have the same prognostic value as a multimarker approach including myoglobin and CK-MB, according to Eggers et al. [84]. In another study of patients with ACS, the combination of cTnT and BNP levels was found of better prognostic value for a composite of death, myocardial infarction, unstable angina and the need for revas- 
Table 1

Biomarkers in cardiovascular disease

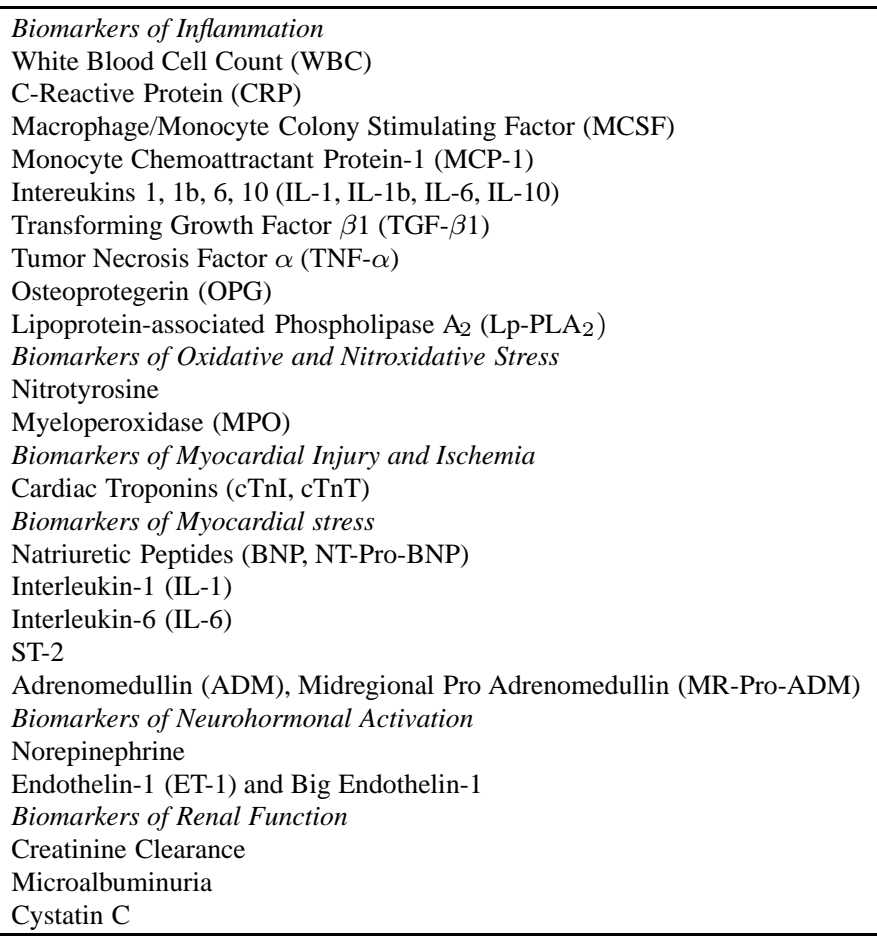

cularization procedures within 30 days than each marker alone. The combination of cTnT and BNP levels showed a sensitivity and relative risk of $78.6 \%(66.2-$ $91)$ and 4.7 (2.3-9.5) respectively for adverse events during follow-up. Moreover, the prognostic value of the combination of cTnT and BNP levels was better than that of the combination of cTnT and CRP levels. Furthermore, the addition of high sensitivity CRP measurement in the panel of cTnT and BNP levels did not add any further prognostic information [85].

Osteoprotegerin (OPG) and BNP levels are increased in acute coronary syndromes. In a recent report by Palazzuoli et al., OPG levels were increased in patients with stable angina compared to controls, however, patients with non ST-elevation acute coronary syndromes had higher OPG levels compared to patients with stable angina $(11.8 \pm 7.1 \mathrm{pmol} / \mathrm{l}, p<0.001)$. The levels of OPG correlated with the number of coronary plaques, whereas BNP correlated with the number of the affected vessels [86]. The findings of the above studies suggest that OPG and BNP may provide complementary information about the atheromatic load and plaque instability in patients with CAD.

In acute coronary syndromes the prognostic value of BNP may be enhanced when combined with the measurements of midregional pro-adrenomedullin (MR-
pro-ADM). Both biomarker measurements were strong predictors of cardiovascular death or heart failure 35 days after the onset of symptoms of the acute event, and MR-pro-ADM provided further risk stratification in patients with increased NT-Pro-BNP levels [87].

Also, the state of renal function, as estimated by creatinine clearance, may further enhance the predictive value of BNP and troponin levels in the risk assessment of patients with acute coronary syndromes. Suwaidi et al. described the prognostic value of renal function for death and myocardial infarction in patients with an acute coronary event at 30- and 180-days. Creatinine clearance was independently associated with risk of mortality (hazard ratio 0.79 in the ST-elevation group and 0.81 in the non-ST-elevation group) and with risk of mortality/MI (hazard ratio 0.93) in the non-STElevation group at 180 days [88].

Mockel et al. evaluated possible combinations between various biomarkers (cardiac troponin I, NTPro-BNP, high sensitivity C-reactive protein, placental growth factor, Lp-PLA 2 , D-dimers, whole blood choline and plasma choline) in patients presenting with an ACS in the emergency department. They found that the combination of NT-Pro-BNP, whole blood choline (WBCHO) and LpPL-A 2 was optimal for risk stratification and superior to other combinations of biomarkers. 
Table 2

Multiple biomarker combinations

\begin{tabular}{ll}
\hline Biomarkers studied & $\begin{array}{l}\text { Associations with outcome inde- } \\
\text { pendent of established risk factors }\end{array}$ \\
\hline Primary Prevention & \\
CRP, IL-6 (Ridker et al.) [68] & CRP, IL-6 \\
CRP, TNF- $\alpha$, IL-6 (Tuomisto et al.) [15] & CRP, TNF- $\alpha$ \\
CRP, IL-6, TNF- $\alpha$ (Cesari et al. [66], Vasan et al. [67]) & CRP, IL-6, TNF- $\alpha$ \\
NT-Pro-BNP, cTn, CRP, Cystatin C (Zethelius et al.) [79] & NT-Pro-BNP, cTn, CRP, Cystatin C \\
& \\
Secondary Prevention & \\
1. Coronary Artery Disease & \\
cTn, WBC (Sabatine et al.) [4] & cTn, WBC \\
CRP, MCSF (Ikonomidis et al.) [13] & CRP, MCSF \\
CRP, MCP-1, BNP (de Lemos et al.) [80] & CRP, MCP-1, BNP \\
CRP, BNP, cTnI (Sabatine et al.) [81] & CRP, BNP, cTnI \\
CRP, IL-6, TNF- $\alpha$, Fibrinogen (Koukkunen et al.) [82] & CRP, IL-6, TNF- $\alpha$, Fibrinogen \\
CRP, cTn, NT-Pro-BNP, CK-MB, Myoglobin (Cameron et al.) [85] & cTn, NT-Pro-BNP \\
CRP, Lp-PLA 2 (Sabatine et al.) [95] & CRP, Lp-PLA ${ }_{2}$ \\
NT-Pro-BNP, OPG (Palazzuoli et al.) [86] & NT-Pro-BNP, OPG \\
BNP, MR-Pro-ADM (Khan et al.) [87] & BNP, MR-Pro-ADM \\
NT-Pro-BNP, IL-6, CRP, Fibrinogen, Cystatin C, Albuminuria (Shlipak et al.) [96] & NT-Pro-BNP, CRP, Albuminuria \\
cTnI, NT-Pro-BNP, CRP, WBCHO, PLCHO, LpPLA ${ }_{2}$, Placental Growth Factor, D-Dimers & NT-Pro-BNP, WBCHO, LpPLA ${ }_{2}$ \\
(Mockel et al.) [89] & \\
Cystatin C, NT-Pro-BNP, Lp-PLA ${ }_{2}$ (Koenig et al.) [94] & Cystatin C, NT-Pro-BNP, Lp-PLA 2 \\
cTn, BNP, MPO, CD40 (Morrow et al.) [91] & cTn, BNP, MPO \\
CRP, $\gamma$ GT, Glucose (Emdin et al.) [93] & CRP, $\gamma$ GT, Glucose \\
2. Heart Failure & \\
BNP, cTn (Fonarow et al.) [101,102] & BNP, cTn \\
BNP, cTn, CRP (Yin et al.) [104] & BNP, cTn, CRP \\
\hline
\end{tabular}

Increased or low concentrations of NT-Pro-BNP and WBCHO identified patients of very high or very low risk for major adverse cardiovascular events (cardiac death, non-fatal myocardial infarction, unstable angina, heart failure requiring admission, urgent PCI and $\mathrm{CABG}$ ) during 42 days of follow-up respectively [89].

Myeloperoxidase (MPO) levels correlate with the prevalence of cardiovascular disease and were found to predict early and late cardiovascular events after an ACS [90]. MPO levels add to the prognostic value of BNP and cTnI regarding short-term cardiovascular effects of patients with non-ST elevation ACS. Patients with elevated baseline MPO ( $>884 \mathrm{pM})$ were at higher risk of non-fatal myocardial infarction or rehospitalization for ACS at 30 days ( 9.3 vs. $4.6 \%, P<0.001)$. In contrast to MPO, soluble CD40 ligand levels were not associated with risk in this population [91].

\section{Chronic Coronary Artery Disease (CAD)}

Studies have shown that there is a synergistic value of MCSF and CRP regarding absolute and relative risk for future cardiovascular events during a 6 year follow-up in patients with chronic CAD [11]. However, it seems that MCSF is a better prognostic marker for short term outcome, whereas CRP predicts cardiovascular events at a longer period of time. This prob- ably reflects cytokine production at different stages of the inflammatory process. Increased levels of TNF- $\alpha$ predict a shorter event-free interval, compared to CRP which predicts long-term cardiovascular events, a fact that suggests a role of CRP in more advanced stages of atherogenesis. Increased levels of CRP and IL-6 in combination to high MCSF or low $\mathrm{TGFb}-1$ predict adverse outcomes in CAD patients independently of traditional risk factors [92]. In the context of the multimarker approach, older biomarkers are re-evaluated, as was done for gamma-glutamyltransferase $(\gamma \mathrm{GT})$, which was found to have an additive prognostic value for mortality to other biomarkers, such as CRP, during a 3-year follow-up period in patients with angiographically documented coronary artery disease [93].

Koenig et al. estimated the incremental value of Lp$\mathrm{PLA}_{2}$ to a model including classical risk factors, renal function as assessed by cystatin $\mathrm{C}$ and hemodynamic stress as assessed by NT-Pro-BNP for cardiovascular events (death, non-fatal MI, stroke) in patients with CAD during 4 years of follow-up. They found that addition of cystatin C and NT-Pro-BNP measurement to the basic model improved its predictive accuracy (Area Under the Curve: 0.71 from 0.69) and when Lp-PLA 2 levels were added the AUC showed a small increase $(0.73$ from 0.71$)$. In the multivariable analyses there 
was a near 2-fold increased risk for future cardiovascular events in patients in the top two tertiles of Lp-PLA 2 mass compared to the lower tertile, after adjustment for markers of inflammation, renal dysfunction and hemodynamic stress [94]. In the PEACE trial, Sabatine et al. showed that elevated Lp-PLA 2 and hs-CRP levels were highly significant predictors of acute coronary syndromes in patients with stable coronary artery disease ( $p<0.005$ and 0.001 respectively), whereas only Lp-PLA $A_{2}$ was a significant predictor for coronary revascularization during 4.8 years days of follow-up [95].

In the Heart and Soul Study the prognostic value of six biomarkers (NT-Pro-BNP, IL-6, CRP, fibrinogen, Cystatin $\mathrm{C}$ and albuminuria) was evaluated in patients with chronic coronary artery disease during an average of 3.5 years of follow up. After multivariable analysis including all biomarkers, NT-Pro-BNP, CRP, and albuminuria were found to retain their prognostic value for cardiovascular events (NT-Pro-BNP Hazard Ratio:1.88, albuminuria Hazard Ratio:1.63, and CRP Hazard Ratio:1.82). Adding all three biomarkers significantly increased the area under the receiver operator curve for the prediction of adverse outcome to 0.77 compared to 0.73 when each biomarker was used on its own [96].

\subsection{Heart failure}

Markers of inflammation are elevated in heart failure. It has been proposed that an initial precipitating event, such as an ischemic cardiac injury, leads to the initiation of inflammatory process via cytokine production, which in turn causes impairment of left ventricular function and accelerates the progression of heart failure [97]. TNF- $\alpha$ and interleukins 1,6, and 10 have been found to be elevated in patients with heart failure [98]. However, the usefulness of interleukins and TNF- $\alpha$ in a multi marker panel as predictive markers in heart failure has not, to our knowledge, been evaluated.

The prognostic value of various neurohormones has been evaluated in the Valsartan Heart Failure Trial (ValHeFT). All the neurohormones in patients with advanced heart failure were significant markers of outcome. BNP was found to be the most powerful predictor of mortality and hospitalization followed by big endothelin-1, norepinephrine, endothelin-1, plasma renin activity, and aldosterone [99].

The natriuretic peptides (BNP and NT-Pro-BNP), biomarkers of elevated intracardiac pressures and myocardial stress, have been shown to add prognostic information about the severity and adverse outcomes in heart failure patients. Supplementary prognostic information to that provided by the natriuretic peptides may be gathered by the measurement of the levels of midregional pro-adrenomedullin (MR-pro-ADM). Khan et al. report that MR-pro-ADM has an additive prognostic value to the levels of NT-Pro-BNP for the development of heart failure after an acute myocardial infarction [84]. However, MR-pro-ADM's predictive value in patients with known heart failure has not been evaluated.

ST2, an interleukin-1 receptor family member, has been studied in patients with heart failure (NYHA class III or IV) and baseline levels correlated with the levels of BNP, Pro-ANP and norepinephrine. Furthermore, after multivariate analysis the change in serum soluble ST2 levels remained significant predictor for mortality or need for heart transplantation, independently of BNP or Pro-ANP levels, suggesting an incremental value of ST2 to BNP or Pro-ANP [100].

Myocardial cell injury and necrosis, as expressed by elevated levels of cardiac troponins, is associated with the progression of heart failure and adverse effects of the disease, as is already well documented. Fonarow et al. have shown that heart failure patients with detectable cardiac troponin I and high BNP levels have a 12-fold increased mortality risk compared to those with both undetectable cardiac troponin I and lower BNP [101]. The investigators of the ADHERE study document that a level of BNP $>840 \mathrm{pg} / \mathrm{mL}$ and increased levels of troponin was associated with a greater mortality risk than $\mathrm{BNP}<840 \mathrm{pg} / \mathrm{mL}$ and normal values of troponin (mortality rate $10.2 \%$ and $2.2 \%$ respectively), showing that admission BNP and cTn levels are significant, independent predictors of in-hospital mortality in acutely decompensated HF [102]. The measurement of high sensitivity troponin $\mathrm{T}$ (hsTnT) in patients with heart failure was evaluated by Latini et al., and it was shown that troponin $\mathrm{T}$ retains a prognostic value at previously undetectable concentrations. cTnT was associated with death and first hospitalization for heart failure (HR:2,08 and 1.55 respectively) and hsTnT was associated with mortality risk (HR:1.05, CI:1.04-1.07 for increments of $0.01 \mathrm{ng} / \mathrm{mL}$ ) [103].

It has been proposed that a multimarker approach including markers of myocardial injury, hemodynamic stress, inflammation and renal function could provide further prognostication than each biomarker alone. Yin et al. propose that the combination of NT-Pro-BNP, cTnI and high sensitivity CRP (hsCRP) have an additive prognostic value to single biomarker measurement in patients with advanced heart failure during a median 
follow-up period of 186 days. Patients with one, two or three elevated biomarkers had a 2.7- $(p=0.125)$, 8.6$(p<0.0001)$ and 23.4- $(p<0.0001)$ fold increase in the risk of adverse events (cardiac death, requirement for heart transplantation or hospitalization for worsening of heart failure) respectively [104].

With the exception of BNP and cTn, little is known about the incremental predictive value of other biomarkers in the risk stratification of patients with heart failure. Several biomarkers of inflammation, myocardial stress, myocardial necrosis, oxidative stress and renal function could be evaluated in a multimarker panel in hope that they may improve risk assessment in heart failure patients and help optimize their treatment.

\section{Conclusion}

Various biomarkers express different pathways and pathophysiologic mechanisms of cardiovascular disease. The work in the field of biomarkers is ongoing and novel biomarkers are being developed and studied constantly. Current thinking supports the notion that the combination of these biomarkers could increase their diagnostic and prognostic value. Research towards this so-called multimarker approach is far from completed and new evidence comes to light as novel biomarkers are being examined. The multimarker approach offers benefits since it allows the study of cardiovascular disease through many different mechanisms, it increases the diagnostic and prognostic information and may help in the design of a strategy for prevention or management of cardiovascular diseases. However, the optimal combination of biomarkers has not been defined yet and further work in this field is needed in order to improve the risk stratification of patients with cardiovascular disease and help physicians optimize their management.

\section{References}

[1] R. Ross, Atherosclerosis - An inflammatory disease, $N$ Engl J Med 340 (1999), 115-126.

[2] R. Bataille and B. Klein, C-reactive protein levels as a direct indicator of interleukin 6 levels in humans in vivo, Arhritis Reum 35 (1992), 982-984.

[3] A.J. Grau, A.W. Boddy, D.A. Dukovic et al., Leukocyte count as an independent predictor of recurrent ischemic events, Stroke 35 (2004), 1147-1152.

[4] M.S. Sabatine, D.A. Morrow, C.P. Cannon et al., Relationship between baseline white blood cell count and degree of coronary artery disease and mortality in patients with acute coronary syndromes: a TACTICS-TIMI 18 substudy, J Am Coll Cardiol 40 (2002), 1761-1768.
[5] P.M. Ridker, J.E. Buring, N.R. Cook and N. Rifai, C-reactive protein, the metabolic syndrome, and risk of incident cardiovascular events: an 8-year follow-up of 14719 initially healthy American women, Circulation 107 (2003), 391-397.

[6] J. Danesh, J.G. Wheeler, G.M. Hirschfield et al., C-reactive protein and other circulating markers of inflammation in the prediction of coronary heart disease, $N$ Engl J Med 250 (2004), 1287-1297.

[7] P.M. Ridker and N. Cook, Clinical usefulness of very high and very low levels of C-reactive protein across the full range of Framingham risk scores, Circulation 109 (2004), 19551959.

[8] C. Heeschen, C.W. Hamm, J. Bruemmer and M.L. Simmons, Predictive value of C-reactive protein and Troponin $\mathrm{T}$ in patients with unstable angina: a comparative analysis. CAPTURE investigators. Chimeric c7E3 AntiPlatelet Therapy in Unstable angina REfractory to standard treatment trial, $J$ Am Coll cardiol 35 (2000), 1535-1542.

[9] P.M. Ridker, C.P. Cannon, D. Morrow et al., C-reactive protein levels and outcomes after statin therapy, $N$ Engl J Med 352 (2005), 20-28.

[10] S.E. Nissen, E.M. Tuzcu, P. Schoenhagen et al., statin therapy, LDL cholesterol, C-reactive protein and coronary artery disease, N Engl J Med 352 (2005), 29-38.

[11] I.S. Anand, R. Latini, V.G. Florea et al., C-reactive protein in heart failure: prognostic value and the effect of valsartan, Circulation 112 (2005), 1428-1434.

[12] I. Ikonomidis, F. Andreotti and P. Nihoyannopoulos, Reduction of daily-life ischaemia by aspirin in patients with angina: underlying link between thromboxane A2 and macrophage colony stimulating factor, Heart 90 (2004), 289-293.

[13] I. Ikonomidis, J. Lekakis, I. Revela, F. Andreotti and P. Nihoyannopoulos, Increased circulating $\mathrm{C}$-reactive protein and macrophage colony stimulating factor are complementary predictors of long-term outcome in patients with chronic coronary artery disease, Eur Heart J 26 (2005), 1618-1624.

[14] B. Levine, J. Kalman, L. Mayer, H.M. Fillit and M. Packer, Elevated circulating levels of tumor necrosis factor in severe chronic heart failure, $N$ Engl J Med 323 (1990), 326-341.

[15] K. Tuomisto, P. Jousilahti, J. Sundvall, P. Pajunen and V. Salomaa, C-reactive protein and tumor necrosis factor as predictors of incident coronary artery and cardiovascular events and total mortality: a population based prospective study, Thromb Haemostat 95 (2006), 511-518.

[16] S. Jono, Y. Ikari, A. Shioi et al., Serum osteoprotegerin levels are associated with the presence and severity of coronary artery disease, Circulation 106 (2002), 1192-1194.

[17] S. Kiechl, G. Schett, G. Wenning et al., Osteoprotegerin is a risk factor for progressive atherosclerosis and cardiovascular disease, Circulation 109 (2004), 2175-2180.

[18] I. Ikonomidis, F. Andreoti, E. Economou, C. Stefanadis, P. Toutouzas and P. Nihoyannopoulos, Increased proinflammatory cytokines in patients with chronic stable angina and their reduction by aspirin, Circulation 100 (1999), 793-798.

[19] M.S. Finkel, R.A. Hoffman, L. Shen, C.V. Oddis, R.L. Simmons and B.G. Hattler, Interleukin 6 as a mediator of stunned myocardium, Am J Cardiol 71 (1993), 1231-1232.

[20] M.C. Deng, M. Erren, A. Lutgen, P. Zimmermann et al., Interleukin 6 correlates with hemodynamic impairment during dobutamine administration in chronic heart failure, Int $J$ Cardiol 57 (1996), 129-134.

[21] I. Ikonomidis, G. Athanassopoulos, J. Lekakis et al., Myocardial ischemia induces interleukin 6 and tissue factor production in patients with coronary artery disease, A 
dobutamine stress echocardiography study, Circulation 112 (2005), 3272-3279.

[22] I. Ikonomidis, G. Athanassopoulos, K. Stamatelopoulos, J. Lekakis et al., Additive prognostic value of interleukin 6 at peak phase of dobutamine stress echocardiography in patients with coronary artery disease, A 6-year follow-up study, Am Heart J 156 (2008), 269-276.

[23] C.J. Packard, D.S. O’Reilly, M.J. Caslake et al., Lipoproteinassociated phospholipase $\mathrm{A}_{2}$ as an independent predictor of coronary heart disease, N Engl J Med 343 (2000), 11481155 .

[24] E.S. Brilakis, J.P. McConnell, R.J. Lennon et al., Association of lipoprotein-associated phospholipase A2 levels with coronary artery disease risk factors, angiographic coronary artery disease, and major adverse events at follow-up, Eur Heart J 26 (2005), 137-144.

[25] E.R. Mohler, C.M. Ballantyne, M.H. Davidson, M. Hanefeld et al., The effect of darapladib on plasma lipoproteinassociated phospholipase $\mathrm{A}_{2}$ activity and cardiovascular biomarkers in patients with stable coronary heart disease or coronary heart disease risk equivalent, $\mathrm{J} \mathrm{Am} \mathrm{Coll} \mathrm{Cardiol} \mathbf{5 1}$ (2008), 1632-1641.

[26] J. Frostegard, A.K. Ulfgren, P. Nyberg et al., Cytokine expression in advanced plaques: dominance of proinflammatory (Th1) and macrophage-stimulating cytokines, Atherosclerosis 145 (1999), 33-43.

[27] A.K.L. Robertson, M. Rudling, X. Zhou, L. Gorelik, A.R. Flavell and G.K. Hansson, Disruption of TGF-beta signaling in T-cells accelerates atherosclerosis, J Clin Invest 112 (2003), 1342-1350.

[28] S. Kojima, P.C. Harpel and D.B. Rifkin, Lipoprotein (a) inhibits the generation of transforming growth factor beta: an endogenous inhibitor of smooth muscle cell migration, $J$ cell Biol 113 (1991), 1439-1445.

[29] E. Amento, N. Ehsani and P. Libby, Cytokines and growth factors positively and negatively regulate interstitial collagen gene expression in human vascular smooth muscle cells, Arterioscl Thromb 11 (1991), 1223-1230.

[30] G. Peluffo and R. Radi, Biochemistry of protein tyrosine nitration in cardiovascular pathology, Cardiovasc Res $\mathbf{7 5}$ (2007), 291-302.

[31] R. Zhang, M.L. Brennan, X. Fu et al., Association between myeloperoxidase levels and risk of coronary artery disease, J Am Med Association 286 (2001), 2136-2142.

[32] V. Nambi, The use of myeloperoxidase as a risk marker for atherosclerosis, Curr Atheroscler Rep 7 (2005), 127-131.

[33] S. Baldus et al., Myeloperoxidase serum level predicts risk in patients with acute coronary syndromes, Circulation $\mathbf{1 0}$ (2003), 1440-1445.

[34] W.H. Tang, M.L. Brennan, K. Philip et al., Plasma myeloperoxidase levels in patients with chronic heart failure, Am J Cardiol 98 (2006), 796-799.

[35] C.A. Henrikson, E.E. Howell, D.E. Bush et al., Prognostic usefulness of marginal Troponin T elevation, Am J Cardiol 93 (2004), 275-279.

[36] L. La Vecchia, G. Mezzena, L. Zanolla et al., Cardiac Troponin $\mathrm{I}$ as diagnostic and prognostic marker in severe heart failure, J Heart Lung Transplant 19 (2000), 644-652.

[37] T.B. Horwich, J. Patel, W.R. MacLellan and G.C. Fonarow, Cardiac Troponin I is associated with impaired hemodynamics, progressive left ventricular dysfunction, and increased mortality rates in advanced heart failure, Circulation $\mathbf{1 0 8}$ (2003), 833-838.
[38] M.P. Hudson, C.M. O'Connor, W.A. Gattis et al., Implications of elevated cardiac Troponin $\mathrm{T}$ in ambulatory patients with heart failure: a prospective analysis, AM Heart J 147 (2004), 546-552.

[39] W.F. Peacock IV, T. De Marco, G.C. Fonarow et al., Cardiac troponin and outcome in acute heart failure, $N$ Engl J Med 358 (2008), 2117-2126.

[40] L.B. Daniels and A.S. Maisel, Natriuretic peptides, J Am Coll Cardiol 50 (2007), 2357-2368.

[41] G.C. Fonarow, W.F. Peacock, C.O. Phillips, M.M. Givertz and M. Lopatin, Admission B-type natriuretic peptide levels and in-hospital mortality in acute decompensated heart failure, J Am Coll Cardiol 49 (2007), 1943-1950.

[42] T. Sugiura, H. Takase, T. Toriyama, T. Goto, R. Ueda and Y. Dohi, Circulating levels of myocardial proteins predict future deterioration of congestive heart failure, J Card Fail 11 (2005), 504-509.

[43] P. Jourdain, G. Jondeau, F. Funck et al., Plasma brain natriuretic peptide-guided therapy to improve outcome in heart failure: the STARS-BNP Multicenter Study, J AM Coll Cardiol 49 (2007), 1733-1739.

[44] D. Logeart, G. Thabut, P. Jourdain et al., Predischarge B-type natriuretic peptide assay for identifying patients at high risk of re-admission after decompensated heart failure, Am Heart J 43 (2004), 635-641.

[45] N. Nagaya, T. Saton, T. Nishikimi et al., Hemodynamic, renal, and hormonal effects of adrenomedullin infusion in patients with congestive heart failure, Circulation 101 (2000), 498-503.

[46] M. Jougasaki, C.M. Wei, L.J. McKinley and J.C. Burnett Jr., Elevation of circulating and ventricular adrenomedullin in human congestive heart failure, Circulation 92 (1995), 286-289.

[47] M. Shimpo, D.A. Morrow, D.O. Weinberg et al., Serum levels of the interleukin-1 receptor family member ST2 predict mortality and clinical outcome in acute myocardial infarction, Circulation 109 (2004), 2186-2190.

[48] J.L. Januzzi Jr., W.F. Peacock, A.S. Maisel et al., Measurement of the interleukin family member ST2 in patients with acute dyspnea: results from the PRIDE (Pro-Brain Natriuretic Peptide Investigation of Dyspnea in the Emergency Department) study, J Am Coll Cardiol 50 (2007), 607-613.

[49] I. Ikonomidis, J.P. Lekakis, M. Nikolaou, I. Paraskevaidis et al., Inhibition of interleukin-1 by anankira improves vascular and left ventricular function in patients with rheumatoid arthritis, Circulation 117 (2008), 2662-2669.

[50] A. Abbate, F.N. Salloum, E. Vecile, A. Das et al., Anakinra, a recombinant interleukin-1 receptor antagonist, inhibits apoptosis in experimental acute myocardial infarction, Circulation 117 (2008), 2670-2683.

[51] J.N. Cohn, T.B. Levine, M.T. Olivari et al., Plasma norepinephrine as a guide to prognosis in patients with chronic congestive heart failure, N Engl J Med 311 (1984), 819-823.

[52] D.L. Moraes, W.S. Colucci and M.M. Givertz, Secondary pulmonary hypertension in chronic heart failure: the role of the endothelium in pathophysiology and management, Circulation 102 (2000), 1718-1723.

[53] M. Hulsmann, B. Stanek, B. Frey et al., Value of cardiopulmonary exercise testing and big endothelin plasma levels to predict short-term prognosis of patients with chronic heart failure, J Am Coll Cardiol 32 (1998), 1695-1700.

[54] M. Hayashi, T. Tsutamoto, A. Wada et al., Immediate administration of mineralocorticoid receptor antagonist spironolactone prevents post-infarct left ventricular remodelling as- 
sociated with suppression of a marker of myocardial collagen synthesis in patients with first anterior acute myocardial infarction, Circulation 107 (2003), 2559-2565.

[55] D.A. Morrow and E. Braunwald, Future of biomarkers in acute coronary syndromes: Moving toward a multimarker strategy, Circulation 108 (2003), 250-252.

[56] J. Arpegard, J. Ostegren, U. De Faire et al., Cystatin C - A marker of peripheral atherosclerotic disease? Atherosclerosis 199 (2008), 397-401.

[57] M.J. Sarnak, R. Katz, C.O. Stehman-Breen et al., Cystatin C concentration as a risk factor for heart failure in older adults, Ann Inter Med 142 (2005), 497-505.

[58] A. Larsson, J. Helmersson, L.O. Hansson and S. Basu, Serum cystatin $\mathrm{C}$ is associated with other cardiovascular risk markers and cardiovascular disease in elderly men, Int J Card $\mathbf{1 2 5}$ (2008), 263-264.

[59] M.G. Shlipak, R. Katz, L.F. Fried et al., Cystatin C and mortality in elderly persons with heart failure, J Am Coll Cardiol 45 (2005), 268-271.

[60] T. Jernberg, B. Lindahl, S. James, A. Larsson, L.O. Hansson and L. Wallentin, Cystatin C: a novel predictor of outcome in suspected or confirmed non-ST-elevation acute coronary syndrome, Circulation 110 (2004), 2342-2348.

[61] K. Wachtell, H. Ibsen, M.H. Olsen et al., Albuminuria and cardiovascular risk in hypertensive patients with left ventricular hypertrophy: the LIFE study, Ann Intern Med 139 (2003), 901-906.

[62] C.T. Valmadrid, R. Klein, S.E. Moss et al., The risk of cardiovascular disease mortality associated with microalbuminuria and gross proteinuria in persons with older-onset diabetes mellitus, Arch Intern Med 160 (2000), 1093-1100.

[63] E.L. Figueiredo, F.V. Leao, L.V. Oliveira et al., Microalbuminuria in nondiabetic and nonhypertensive systolic heart failure patients, Congest Heart Fail 14 (2008), 234-238.

[64] E. Ingelsson, J. Sundstrom, L. Lind et al., Low-grade albuminuria and the incidence of heart failure in a communitybased cohort of elderly men, Eur Heart J 28 (2007), 17391745 .

[65] K.L. Margolis, J.E. Manson, P. Greenland et al., Leukocyte count as a predictor of cardiovascular events and mortality in postmenopausal women: The Women's Health Initiative Observational Study, Arch Intern Med 165 (2005), 500-508.

[66] M. Cesari, B.W. Penninx, A.B. Newman et al., Inflammatory markers and onset of cardiovascular events: Results from the Health ABC study, Circulation 108 (2003), 2317-2322.

[67] R.S. Vasan, L.M. Sullivan, R. Roubenoff et al., Inflammatory markers and risk of heart failure in elderly subjects without prior myocardial infarction: the Framingham Heart Study, Circulation 107 (2003), 1486-1491.

[68] P.M. Ridker, N. Rifai, M.J. Stampfer and C.H. Hennekens, Plasma concentration of IL-6 and the risk of future myocardial infarction among apparently healthy men, Circulation 101 (2000), 1767-1772.

[69] L.S. Rallidis, M.G. Zolidaki, H.S. Manioudaki, N.P. Laoutaris, A.H. Velissaridou and E.G. Papasteriadis, Prognostic value of $\mathrm{C}$-reactive protein, fibrinogen, interleukin 6 and macrophage colony stimulating factor in severe unstable angina, Clin Cardiol 25 (2002), 461-466.

[70] A.R. Folsom, L.E. Chambless, C.M. Ballantyne et al., An assessment of incremental risk prediction using $\mathrm{C}$-reactive protein and other novel risk biomarkers: the atherosclerosis risk in communities study, Arch Intern Med 166 (2006), 1368-1373.
[71] P.M. Ridker, E. Danielson, F.A. Fonseca, J. Genest et al., Rosuvastatin to prevent vascular events in men and women with elevated C-reactive protein, $N$ Engl J Med 359 (2008), 2195-2207.

[72] C. Kistorp, I. Raymond, F. Pederson, F. Gustafsson, J. Faber and P. Hildebrandt, N-Terminal pro brain natriuretic peptide, $\mathrm{C}$-reactive protein, and urinary albumin levels as predictors of mortality and cardiovascular events in older adults, JAMA 6 (2005), 1609-1616.

[73] T. Suzuki, D. Hayashi, T. Yamazaki et al., Elevated B-type natriuretic peptide levels after anthracyclin administration, Am Heart J 136 (1998), 362-363.

[74] S. Blankenburg, M.J. McQueen, M. Smieja et al., HOPE Study investigators, Comparative impact of multiple biomarkers and $\mathrm{N}$-terminal pro-brain natriuretic peptide in the context of conventional risk factors for the prediction of recurrent cardiovascular events in the Heart Outcomes Prevention Evaluation (HOPE) Study, Circulation 114 (2006), 201-208.

[75] P. Bogarty, J.M. Brophy, L. Boyer et al., Fluctuating inflammatory markers in patients with stable ischemic heart disease, Arch Intern Med 165 (2005), 221-226.

[76] C. De Filippi, S. Wasserman, S. Rosanio et al., Cardiac Troponin $\mathrm{T}$ and $\mathrm{C}$-reactive protein for predicting prognosis, coronary atherosclerosis, and cardiomyopathy in patients undergoing long-term hemodialysis, JAMA 290 (2003), 353-359.

[77] F.S. Apple, M.M. Murakami, L.A. Pearce and C.A. Herzog, Multi-biomarker risk stratification of $\mathrm{N}$-terminal pro-B-type natriuretic peptide, high sensitivity $\mathrm{C}$-reactive protein, and cardiac Troponin $\mathrm{T}$ and $\mathrm{I}$ in end-stage renal disease for allcause death, Clin Chem 50 (2004), 2279-2285.

[78] T.J. Wang, G. Philimon, M.G. Larson, G.H. Tofler et al., Multiple biomarkers for the prediction of first major cardiovascular events and death, N Engl J Med 355 (2006), 26312639.

[79] B. Zethelius, L. Berglund, J. Sundstrom et al., Use of multiple biomarkers to improve the prediction of death from cardiovascular causes, $N$ Engl J Med 358 (2008), 2107-2116.

[80] J.A. De Lemos, D.A. Morrow, M.A. Blazing, P. Jarolim, S.D. Wiviott et al., Serial measurement of monocyte chemoattractant protein-1 after acute coronary syndromes. Results from the A to Z trial, J Am Coll Cardiol 50 (2007), 2117-2124.

[81] M.S. Sabatine, D.A. Morrow, J.A. De Lemos et al., Multimarker approach to risk stratification in non-ST elevation acute coronary syndromes: simultaneous assessment of troponin-I, C-reactive protein and B-type natriuretic peptide, Circulation 105 (2002), 17760-17763.

[82] H. Koukkunen, K. Penttila, A. Kemppainen et al., C-reactive protein, fibrinogen, interleukin-6 and tumor necrosis factoralpha in the prognostic classification of unstable angina pectoris, Ann Med 33 (2001), 37-47.

[83] S.D. Wiviott,C.P. Cannon, D.A. Morrow et al., Differential expression of cardiac biomarkers by gender in patients with unstable angina/non-ST-elevation myocardial infarction: a TACTICS-TIMI-18 (Treat Angina with Aggrastat and determine Cost of Therapy with an Invasive or Conservative Strategy-Thrombolysis In Myocardial Infarction 18) substudy, Circulation 109 (2004), 580-586.

[84] K.M. Eggers, J. Oldgren, A. Nordenskjold and B. Lindahl, Combining different biochemical markers of myocardial ischemia does not improve risk stratification in chest pain patients compared to troponin alone, Coron Art Dis 16 (2005), 315-319. 
[85] S.J. Cameron, L.J. Sokoll, O.F. Laterza et al., A multi-marker approach for the prediction of adverse events in patients with acute coronary syndromes, Clin Chim Acta 376 (2007), 168173.

[86] A. Palazzuoli, V. Rizzello, A. Calabro, M. Gallotta et al., Osteoprotegerin and B-type natriuretic peptide in non-ST elevation acute coronary syndromes: relation to coronary artery narrowing and plaques number, Clin Chim Acta 391 (2008), 74-79.

[87] S.Q. Khan, R.J. O’Brien, J. Struck et al., Prognostic value of midregional pro-adrenomedullin in patients with acute myocardial infarction: the LAMP (Leicester Acute Myocardial Infarction Peptide) study, J Am Cardiol 49 (2007), 15251532 .

[88] A.J. Suwaidi, D.N. Reddan, K. Williams et al., Prognostic implications of abnormalities in renal function in patients with acute coronary syndromes, Circulation 106 (2002), 974-980.

[89] M. Mockel, O. Danne, R. Muller, J.O. Vollert et al., Development of an optimized biomarker strategy for early risk assessment of patients with acute coronary syndromes, Clin Chim Acta 393 (2008), 103-109.

[90] E. Cavusoglu, C. Ruwende, E. Calvin, V. Chopra, S. Yanamadala et al., Usefulness of baseline plasma myeloperoxidase levels as an independent predictor of myocardial infarction at two years in patients presenting with acute coronary syndrome, J Am Cardiol 99 (2007), 1364-1368.

[91] D.A. Morrow, M.S. Sabatine, M.L. Brennan, J.A. deLemos et al., Concurrent evaluation of novel cardiac biomarkers in acute coronary syndrome: Myeloperoxidase and soluble CD40 ligand and the risk of recurrent ischemic events in TACTICS-TIMI 18, Eur Heart J 28 (2008), 1096-1112.

[92] I. Ikonomidis, J. Lekakis, K. Stamatelopoulos, G. Vamvakou and D. Kremastinos, Inflammatory and non-invasive markers: The multimarker approach for risk stratification in coronary artery disease, Atherosclerosis 199 (2008), 3-11.

[93] M. Emdin, C. Passino, C. Michelassi, L. Donato, A. Pompella and A. Paolicchi, Additive prognostic value of gammaglutamyltransferase in coronary artery disease, Int $J$ Card (2008), (article in press).

[94] W. Koenig, D. Twardella, H. Brenner and D. Rothenbacher, Lipoprotein-associated phospholipase $\mathrm{A}_{2}$ predicts future cardiovascular events in patients with coronary heart disease independently of traditional risk factors, markers of inflammation, renal function, and hemodynamic stress, Arterioscl Thromb Vasc Biol 26 (2006), 1586-1593.

[95] M.S. Sabatine, D.A. Morrow, M. O’Donoghue, K.A. Jablonski et al., Postic utility of lipoprotein-associated phospholipase A2 for cardiovascular outcomes in patients with stable coronary artery disease, Arterioscl Thromb Vasc Biol 27 (2007), 2463-2469.

[96] M.G. Shlipak, J.H. Ix, K. Bibbins-Doming, F. Lin and M.A. Whooley, Biomarkers to predict recurrent cardiovascular disease: the Heart And Soul Study, Amer J Med 121 (2008), 50-57.

[97] Y. Seta, K. Shan, B. Bozkurt, H. Oral and D.L. Mann, Basic mechanisms in heart failure: the cytokine hypothesis, J Card Fail 2 (1996), 243-249.

[98] S.D. Anker and S. von Haethling, Inflammatory mediators in chronic heart failure: an overview, Heart 90 (2004), 464470.

[99] R. Latini, S. Masson, I. Anand et al., The comparative prognostic value of plasma neurohormones at baseline in patients with heart failure enrolled in Val-HeFT, Eur Heart $J$ 25 (2004), 292-299.

[100] E.O. Weinberg, M. Shimpo, S. Hurwitz et al., Identification of serum soluble ST2 receptor as a novel heart failure biomarker, Circulation 107 (2003), 721-726.

[101] G.C. Fonarow and T.B. Horwich, Combining natriuretic peptides and necrosis markers in determining prognosis in heart failure, Rev Cardiovasc Med 4 (2003), 20-28.

[102] G.C. Fonarow, W.F. Peacock, T.B. Horwich et al., Usefulness of B-type natriuretic peptide and cardiac troponin levels to predict in-hospital mortality from ADHERE, Am J Cardiol 101 (2008), 231-237.

[103] R. Latini, S. Masson, I.S. Anand et al., Prognostic value of very low plasma concentrations of troponin $\mathrm{T}$ in patients with stable chronic heart failure, Circulation 116 (2007), 12421249.

[104] W.H. Yin, J.W. Chen, A.N. Feng et al., Multimarker approach to risk stratification among patients with advanced chronic heart failure, Clin Cardiol 30 (2007), 397-402. 


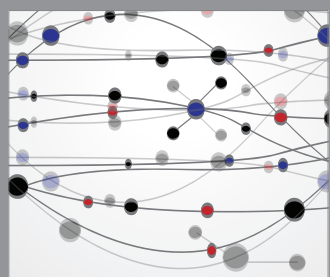

The Scientific World Journal
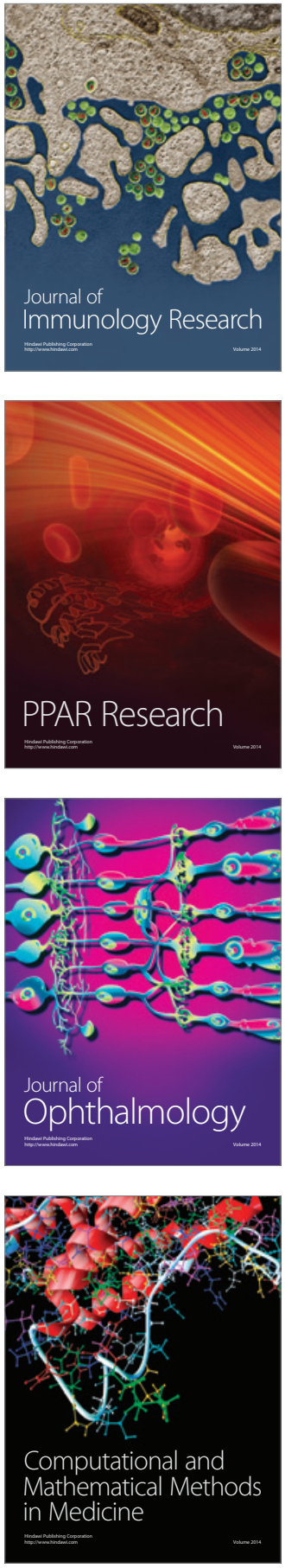

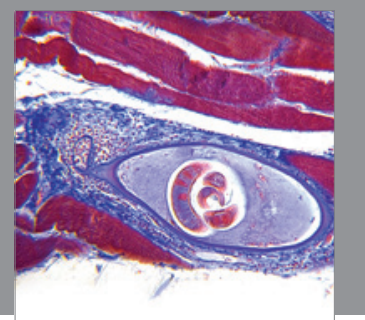

Gastroenterology

Research and Practice
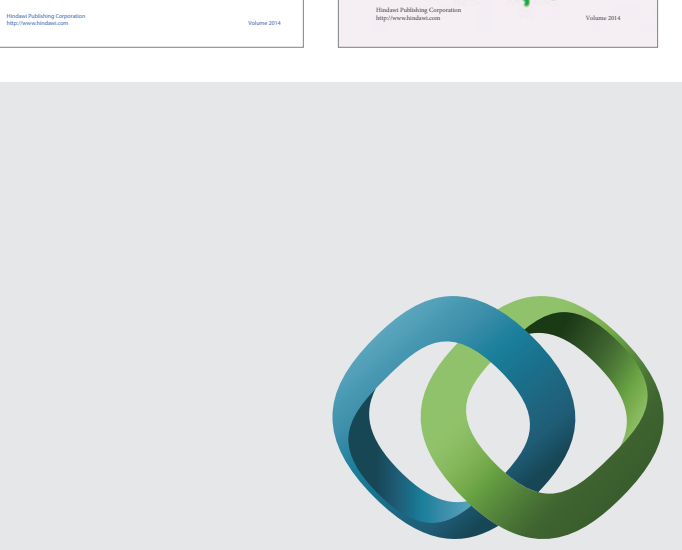

\section{Hindawi}

Submit your manuscripts at

http://www.hindawi.com
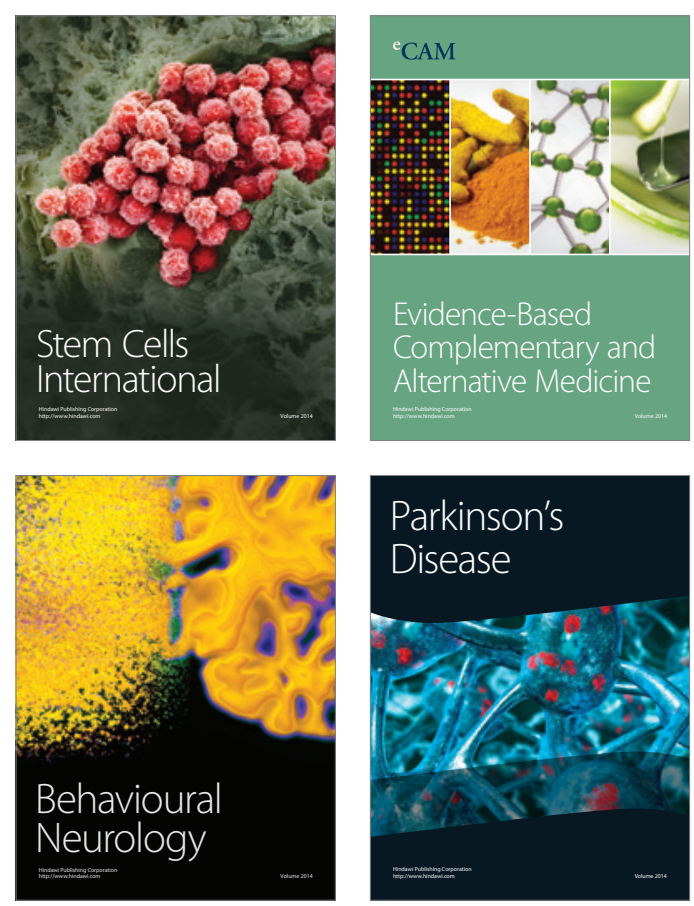

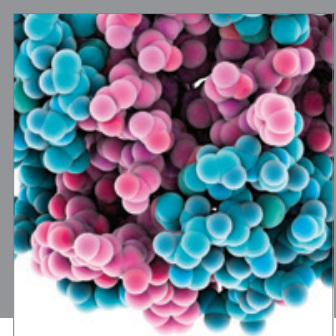

Journal of
Diabetes Research

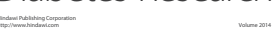

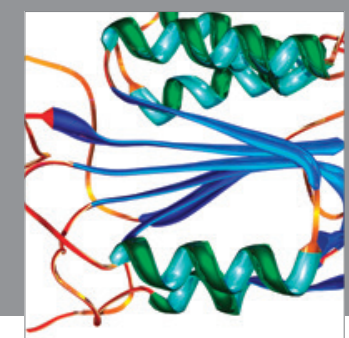

Disease Markers
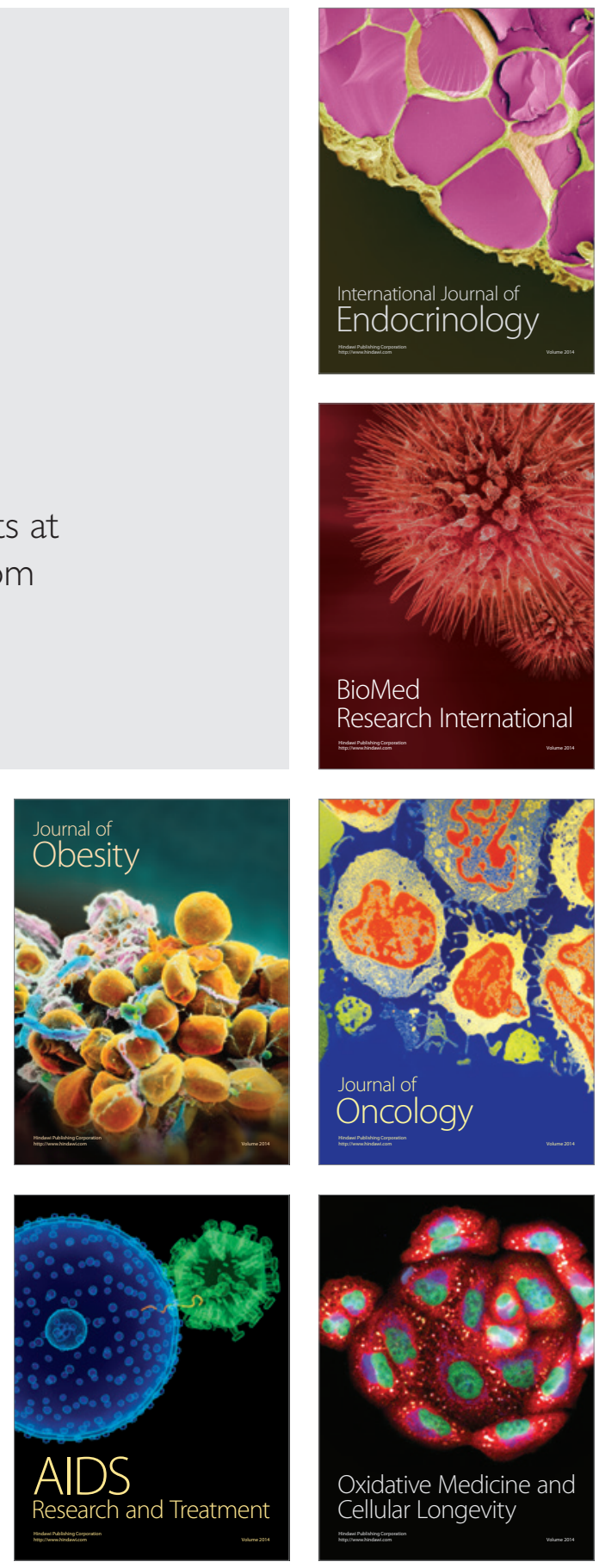